\title{
Landscape of transcription termination in Arabidopsis revealed by single-molecule nascent RNA sequencing
}

Weipeng Mo ${ }^{1,2,3 \dagger}$, Bo Liu ${ }^{1,2,3 \dagger}$, Hong Zhang ${ }^{1,2,3}$, Xianhao Jin ${ }^{1,2,3}$, Dongdong Lu ${ }^{1,2,3}$, Yiming Yu ${ }^{1,2,3}$, Yuelin Liu ${ }^{1,2,3}$, Jinbu Jia ${ }^{1,2,3}$, Yanping Long ${ }^{1,2,3}$, Xian Deng ${ }^{4}$, Xiaofeng Cao ${ }^{4}$, Hongwei Guo ${ }^{1,2,3}$ and Jixian Zhai ${ }^{1,2,3^{*}}$

\author{
* Correspondence: zhaijx@sustech. \\ edu.cn \\ 'Weipeng Mo and Bo Liu \\ contributed equally to this work. \\ ${ }^{1}$ Department of Biology, School of \\ Life Sciences, Southern University of \\ Science and Technology, Shenzhen \\ 518055, China \\ ${ }^{2}$ Institute of Plant and Food \\ Science, Southern University of \\ Science and Technology, Shenzhen \\ 518055, China \\ Full list of author information is \\ available at the end of the article
}

\begin{abstract}
Background: The dynamic process of transcription termination produces transient RNA intermediates that are difficult to distinguish from each other via short-read sequencing methods.

Results: Here, we use single-molecule nascent RNA sequencing to characterize the various forms of transient RNAs during termination at genome-wide scale in wildtype Arabidopsis and in atxrn3, fpa, and met1 mutants. Our data reveal a wide range of termination windows among genes, ranging from $\sim 50 \mathrm{nt}$ to over $1000 \mathrm{nt}$. We also observe efficient termination before downstream tRNA genes, suggesting that chromatin structure around the promoter region of tRNA genes may block pol II elongation. 5' Cleaved readthrough transcription in atxrn3 with delayed termination can run into downstream genes to produce normally spliced and polyadenylated mRNAs in the absence of their own transcription initiation. Consistent with previous reports, we also observe long chimeric transcripts with cryptic splicing in fpa mutant; but loss of CG DNA methylation has no obvious impact on termination in the met1 mutant.
\end{abstract}

Conclusions: Our method is applicable to establish a comprehensive termination landscape in a broad range of species.

Keywords: Transcription termination, Nascent RNA, Nanopore sequencing, Arabidopsis

\section{Background}

Transcription termination is the critical final step in RNA synthesis, during which nascent RNA is released from the complex of RNA Polymerase II (Pol II) and DNA template [1]. Termination is essential to prevent uncontrolled readthrough transcription from invading downstream genes [2,3]. Decades of extensive research in this area has proposed the allosteric/antiterminator model [4], the torpedo model [5], and later a unified model that combines these two to explain the mechanism of polyadenylation signal (PAS)-dependent termination [1, 3, 6-8]. In mammalian cells, after Pol II passed

(c) The Author(s). 2021 Open Access This article is licensed under a Creative Commons Attribution 4.0 International License, which permits use, sharing, adaptation, distribution and reproduction in any medium or format, as long as you give appropriate credit to the original author(s) and the source, provide a link to the Creative Commons licence, and indicate if changes were made. The images or other third party material in this article are included in the article's Creative Commons licence, unless indicated otherwise in a credit line to the material. If material is not included in the article's Creative Commons licence and your intended use is not permitted by statutory regulation or exceeds the permitted use, you will need to obtain permission directly from the copyright holder. To view a copy of this licence, visit http://creativecommons.org/licenses/by/4.0/. The Creative Commons Public Domain Dedication waiver (http://creativecommons.org/publicdomain/zero/1.0/) applies to the data made available in this article, unless otherwise stated in a credit line to the data. 
through the poly(A) site, cleavage and polyadenylation process is initiated, and transcription continues until Pol II disassociated from the DNA template and nascent RNA released $[2,6]$. The $3^{\prime}$ end of mature mRNA is determined by the cleavage event, rather than the site where transcription terminates. The cleavage at poly(A) site provides an entry for $5^{\prime} \rightarrow 3^{\prime}$ exonuclease (Xrn2 in human, Rat1 in yeast, AtXRN2/3 in Arabidopsis) to degrade the 3 ' cleavage product that is still presumably being synthesized by Pol II $[6,9-11]$. The readthrough transcripts accumulate in mutants with a defective cleavage and polyadenylation complex [12,13] or a loss-of-function exonuclease $[9,14,15]$. The kinetic competition between Pol II and exonuclease promotes termination [1, 2, 16]. As a result, readthrough transcription can continue for up to a few thousand nucleotides (nt) downstream of the poly(A) site $[17,18]$. Since the Arabidopsis genome is highly compact, with $\sim 38,000$ genes concentrated in only $\sim 135 \mathrm{Mb}$ DNA sequence [19], termination is essential to prevent the transcriptional collisions between flanking genes. To date, the pattern of Pol II termination in plants remains largely unknown. Thus, we set out to study transcription termination in the model plant Arabidopsis.

The majority of the termination RNA intermediates are transient and rapidly degraded after cleavage, and are therefore difficult to capture and characterize. Several short-read Illumina-based methods have been developed to analyze nascent RNAs [20], but their aims are primarily on the elongating fraction of RNAs before termination, rather than the readthrough and cleaved ones. GRO-seq [21] and PRO-seq [22] performed a run-on with isolated nuclei, which might compromise the termination machinery. NET-seq [23], mNET-seq [24], and POINT-seq [25] require immunoprecipitation of Pol II, which might miss terminating RNAs that are no longer associated with Pol II, such as the cleaved and polyadenylated pre-mRNAs after Pol II passing through the poly(A) site, and cannot distinguish between individual peaks caused by Pol II accumulation and co-transcriptional cleavage [20-24]. TT-seq [17] was indeed able to define transcription termination sites, but due to the limited read length of Illumina sequencing and the RNA fragmentation step during library construction, it also cannot distinguish whether the readthrough transcripts are cleaved or not. Therefore, the field of transcription termination would benefit from a new high-throughput method that can capture and distinguish the various forms of RNA intermediates.

Recent advances in third-generation long-read sequencing technologies, such as PacBio and Nanopore [26, 27], have enabled the detection of full-length mRNAs or cDNAs [28-32]. In particular, the Oxford Nanopore direct RNA sequencing (ONT DRS) can recognize and distinguish various forms of RNA modifications that cause unique changes of current when RNA molecule is passing through the nanopore [26]. The applications of long-read sequencing (LRS) in characterizing full-length nascent RNAs have revealed co-transcriptional splicing kinetics in a variety of species by simultaneously tracking splicing status and position of Pol II elongation on the same RNA molecule, such as nano-COP in human and drosophila [33, 34], LRS of nascent RNA in yeast and mouse [35, 36], POINT-nano in human [25], and recent work from our group in Arabidopsis [37]. Here, we demonstrate the application of single-molecule nascent RNA sequencing in studying transcription termination by simultaneously recording Pol II readthrough distance, cleavage status, and polyadenylation on the same RNA molecule, and revealed the global landscape of transcription termination in Arabidopsis. 


\section{Results}

Single-molecule nascent RNA sequencing captures RNA intermediates during termination Our group recently developed a single-molecule nascent RNA sequencing method named FLEP-seq (Full-Length Elongating and Polyadenylated RNA sequencing) [38] by using the chromatin-bound nascent RNA to study the coordination between Pol II elongation and splicing [37]. Here, we demonstrate that the comprehensive profile of full-length nascent RNAs in Arabidopsis captured by FLEP-seq can be applied to study transcription termination by characterizing the various forms of RNA intermediate produced during the termination process (Fig. 1a). Compared to previous short-read Illumina-based methods, the long-read Nanopore sequencing of FLEP-seq enables us to distinguish the full-length readthrough RNAs from the 5' and 3' cleaved transient RNAs (Fig. 1a), which are generated by the 3 '-end processing factors at the poly(A) site [7, 43]. FLEP-seq also captures the nascent polyadenylated mRNAs that are still associated with chromatin, thus allow us to obtain the precise location of poly(A) site for each gene [30, 44] (Additional file 1, Fig. S1a-c). Given the heterogeneity of poly(A) sites at Arabidopsis 3' UTRs [19, 45], having both the poly $(\mathrm{A})$ site information and the cleavage information in the same library can facilitate our analysis by enabling a more accurate assessment of cleavage events.

The advantage of having the unfractionated long RNAs from FLEP-seq data enabled us to distinguish and categorize the RNA intermediates into four groups: readthrough transcripts, 5' cleavage products, 3' cleavage products, and poly(A) transcripts (Fig. 1a; Additional file 1, Fig. S2). Take DRM2 (AT2G33830) as an example (Fig. 1b, upper panel), readthrough transcripts are those that extended downstream of the poly(A) site; 5 ' cleavage products are those cleaved at the poly(A) site but not yet polyadenylated; 3 ' cleavage products are full-length transcripts with their $5^{\prime}$ ends located downstream of the poly $(\mathrm{A})$ site; and poly(A) transcripts are RNAs with poly(A) tails, and this result is also used to define the position of poly(A) site. Previous short-read based nascent RNA sequencing methods, such as pNET-seq, plaNET-seq, and GRO-seq have been applied in Arabidopsis [39-42] and in other plants including cassava and maize [46], are mostly developed for detecting Pol II-associated elongating RNAs, and can also detect RNA signal downstream of poly(A) site (Fig. 1b, lower panel). In particular, the pNET-seq method can detect nascent RNAs that are associated with various isoforms of Pol II CTD, including total CTD, unphosphorylated, phosphorylation on Ser 2 and Ser 5 [41], and thus may distinguish Pol II at certain transcription step. Compared to these methods, our FLEP-seq method captures a much more comprehensive and smoother pattern of termination and has the unique advantage of distinguishing whether the RNA intermediates are cleaved or not (Fig. 1b). Moreover, in the cases where two neighboring genes are immediately adjacent to each other, short-read-based methods would have difficulty assigning the reads near gene boundaries, while Nanopore long reads from adjacent genes can be easily separated (Fig. 1e).

Genome-wide analysis showed that the poly(A) transcripts are the most abundant forms of the four, followed by readthrough transcripts and 5 ' cleavage products (Fig. 1c, d). The 3' cleavage products only occupied a low proportion (1\%) among the RNA intermediates, consistent with the rapid degradation of uncapped transcripts by exonuclease AtXRN3 in Arabidopsis [14, 47, 48]. FLEP-seq monitors both cotranscriptional cleavage and readthrough, whereas other methods do not. Therefore, by 


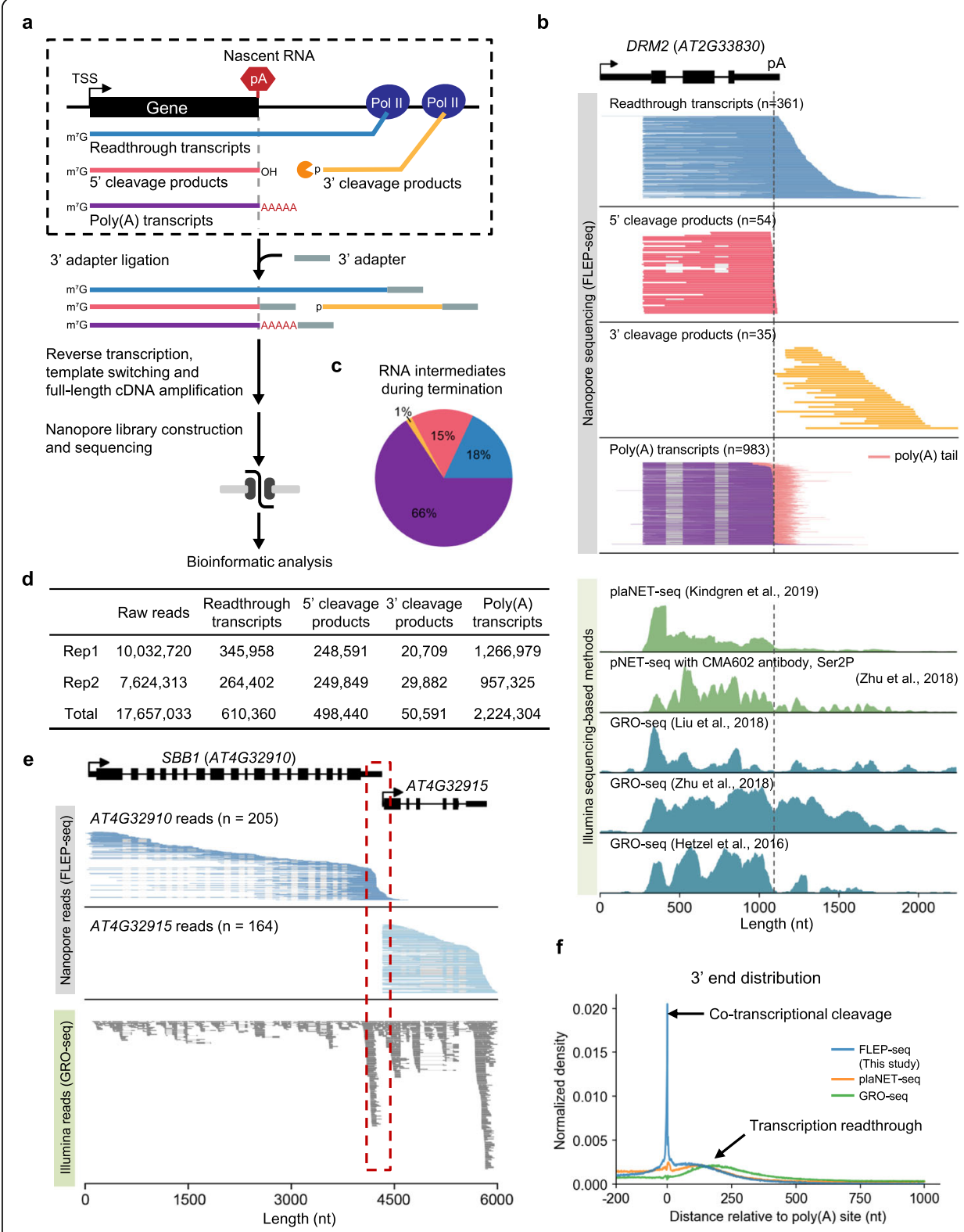

Fig. 1 Single-molecule Nascent RNA Sequencing (FLEP-seq) captures various transient RNA intermediates produced during transcription termination. a Schematic of the FLEP-seq library preparation and sequencing. A $3^{\prime}$ adapter is ligated to nascent RNA for reverse transcription and full-length CDNA cloning. $\mathbf{b}$ Example of the RNA intermediates from gene DRM2. Upper panel: Nanopore reads are aligned to gene ordered by $3^{\prime}$ end position. The number of individual long reads $(n)$ is indicated. Lower panel: coverage of Illumina shortread nascent RNA data from previous studies [39-42]. The gray dash line indicates the poly(A) site (pA). c Proportion of various RNA intermediates during termination. Readthrough transcripts (blue), $5^{\prime}$ cleavage products (red), 3' cleavage products (yellow) and poly(A) transcripts (purple). d Sequencing summary for two biological replicates of FLEP-seq libraries in wildtype Col-0. e Advantage of Nanopore (upper) over Illumina [40] (lower) in separating reads from closely adjacent genes. The red dashed box highlights the intergenic region. $\mathbf{f}$ Meta-profile showing the $3^{\prime}$ end distribution of non-poly $(A)+$ nascent RNA detected by the FLEP-seq, plaNET-seq [42] reads, and GRO-seq [40] near poly(A) site 
analyzing the $3^{\prime}$ end of the non-poly(A) RNAs, our results showed a clear pattern for termination: with a sharp peak enriched at the poly(A) site followed by a gradual decline (Fig. 1f). Taken together, the full-length nascent RNA sequencing method is well suited for tracking various transient RNA products during transcription termination.

\section{The termination landscape of Arabidopsis genes}

Transcription termination of Pol II is thought to be a random process that occurs at different distances downstream of the poly(A) site $[18,49]$. We refer to the distance in which Pol II traveled past the poly(A) site before released from DNA as the previously described "termination window (TW)" [15, 17], also known as "termination zone" [18, 50]. To date, the genome-wide measurement of termination window has only been accessed by short-read sequencing, including in human cells with TT-seq method [17], in yeast with 4tU-seq method [15], and in Arabidopsis with plaNET-seq method [42]. The TT-seq study found a wide range of termination windows among $~ 7000$ genes in human cells, with a median width of $\sim 3300$ bp and can go up to over $10 \mathrm{~kb}$ [17]. The $4 \mathrm{tU}$-seq study found that the median termination window is $163 \mathrm{bp}$ in yeast [15]. The plaNET-seq study suggested a median readthrough distance of $\sim 500 \mathrm{bp}$ for Arabidopsis genes [42]. Compared to these methods, our FLEP-seq method can capture and distinguish the various forms of full-length terminating RNAs with unprecedented depth and resolution, thus presents an opportunity to examine termination patterns for individual genes in detail. For each nascent RNA molecule, readthrough can be calculated as the distance between its 3' end of the RNA and the poly(A) site of the gene (Fig. 2a). Because the detection of the longest readthrough RNA at any loci will be correlated with sequencing depth, using the longest readthrough distance to represent the termination window will make the estimation sensitive to fluctuations of sequencing depth (Fig. 1d, Fig. 2b, c). Our single-molecule long-read method can track each RNA molecule separately, therefore enabling us to use the median readthrough distance of each gene to represent its termination window size as a more robust measurement (Fig. 2b, d). We observed consistent TW size (Pearson's $R=0.91$ ) for individual genes between the two biological replicates of FLEP-seq libraries (Fig. 2d). Hence, we use the median readthrough distance to represent the TW size in the following analyses. We found the lengths of TWs vary drastically among the 9830 Arabidopsis genes analyzed (each with a minimal of 15 readthrough reads), ranging from $\sim 50$ nt to over $1000 \mathrm{nt}$ (Fig. 2e), with the median size at $~ 160 \mathrm{nt}$ (Additional file 2, Table S1). For example, the termination of gene $A T 3 G 51730$ occurred quickly downstream of the poly(A) site with a TW size of $85 \mathrm{nt}$ (Fig. 2f), while gene AT1G62820 (CML14) has many longer readthrough transcripts with a TW size of $686 \mathrm{nt}$ (Fig. 2g). Even using the longest readthrough to represent TW, the median of which is $521 \mathrm{nt}$, the TW in Arabidopsis is still considerably shorter than the TW in human reported by TT-seq [17]. The much shorter termination windows of Arabidopsis compared to human may be due to the compact genome size of Arabidopsis, which is $\sim 20$ times smaller, and hence has a much denser gene arrangement. Taken together, our data reveals a comprehensive termination landscape of Arabidopsis.

\section{Pol II co-localizes with termination factors at the end of termination window}

The transition from transcription elongation to termination requires the slow down or pause of the elongating Pol II [51]. The pausing of Pol II at the $3^{\prime}$ end of the gene is usually associated with Pol II carboxy-terminal domain (CTD) serine 2 phosphorylation 


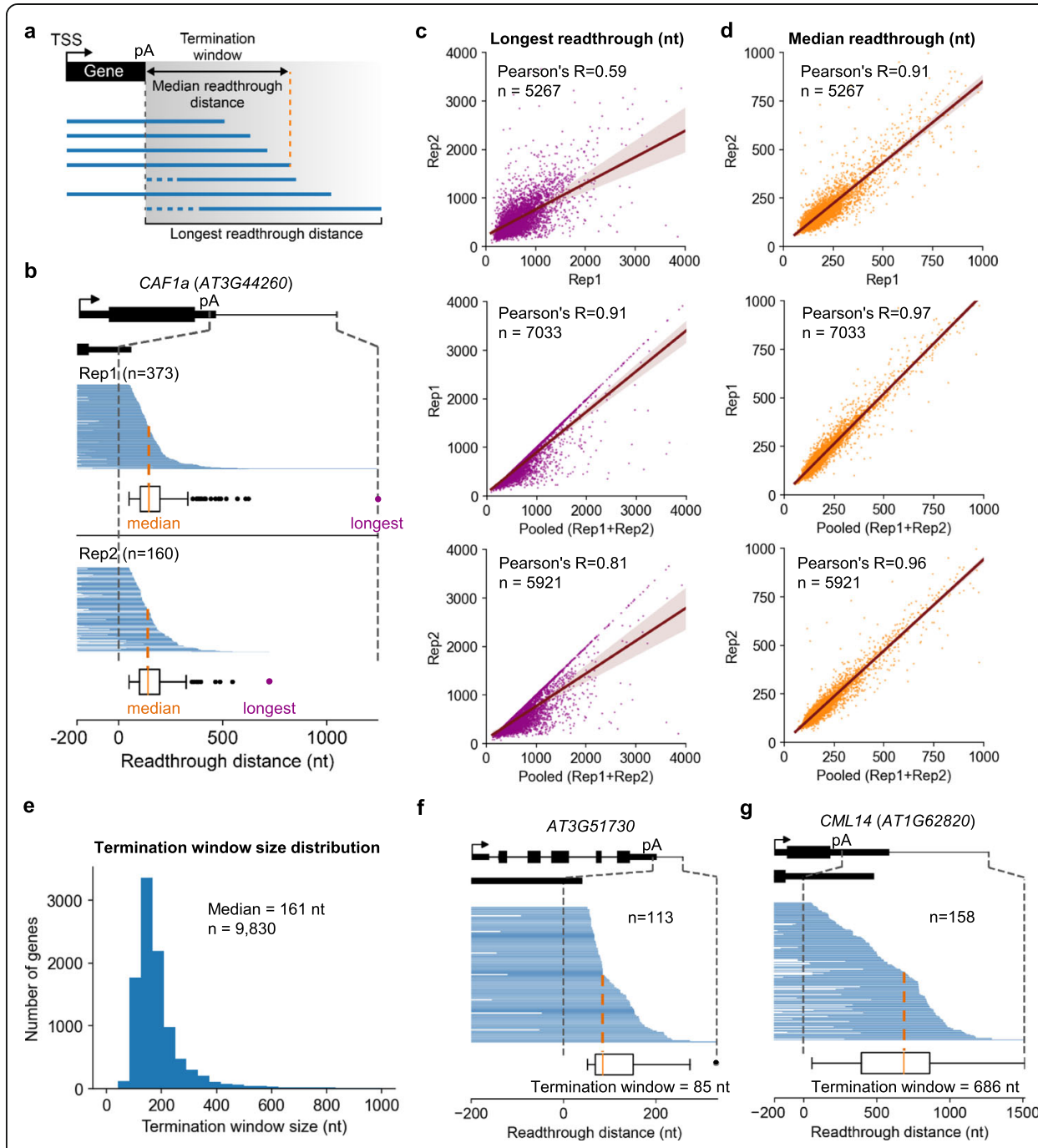

Fig. 2 The termination landscape in Arabidopsis. a Schematic diagram shows the definition of termination window presented in this study. Reads with $3^{\prime}$ end located more than 50 nt downstream of poly(A) site are used to define termination window. The readthrough distance is calculated as the distance between the poly(A) site and the $3^{\prime}$ end of reads. The median readthrough distance at the corresponding gene is used to represent its termination window size. $\mathbf{b}$ Comparison of termination window size quantified by either the median or longest readthrough distance. The gene model is shown at the top and is zoom-in to highlight the region from $200 \mathrm{nt}$ upstream of the poly(A) site to the longest readthrough distance. The boxplots show the readthrough distance distribution for all reads in the region. pA, poly(A) site (also in Fig. 2f, g, and Fig. 4a, d). c, d Scatter plots for the longest (c) and median (d) readthrough distance per gene between replicates (a minimum of 15 readthrough reads for calculating the termination window size). The Pearson's correlation coefficient and the number of genes $(n)$ are shown. e The distribution of termination window size among genes. $\mathbf{f}, \mathbf{g}$ Examples of genes with short and long termination windows, respectively

(Ser2P) [2, 41]. Previous studies revealed that the Pol II accumulation downstream of poly(A) site is related to the $3^{\prime}$ pausing $[18,50,52]$. Compared to previously published ChIP-seq data [53], we observed that ends of the termination window precisely reside with the peaks of Pol II and Ser2P, further suggesting that using median instead of the longest readthrough distance to represent $\mathrm{TW}$ is a more appropriate measurement (Fig. 3a). The presence of termination window engaged Pol II peak could also be observed in pNET-seq [41] and GRO-seq [40] data (Fig. 3b; Additional file 1, Fig. S3a). 


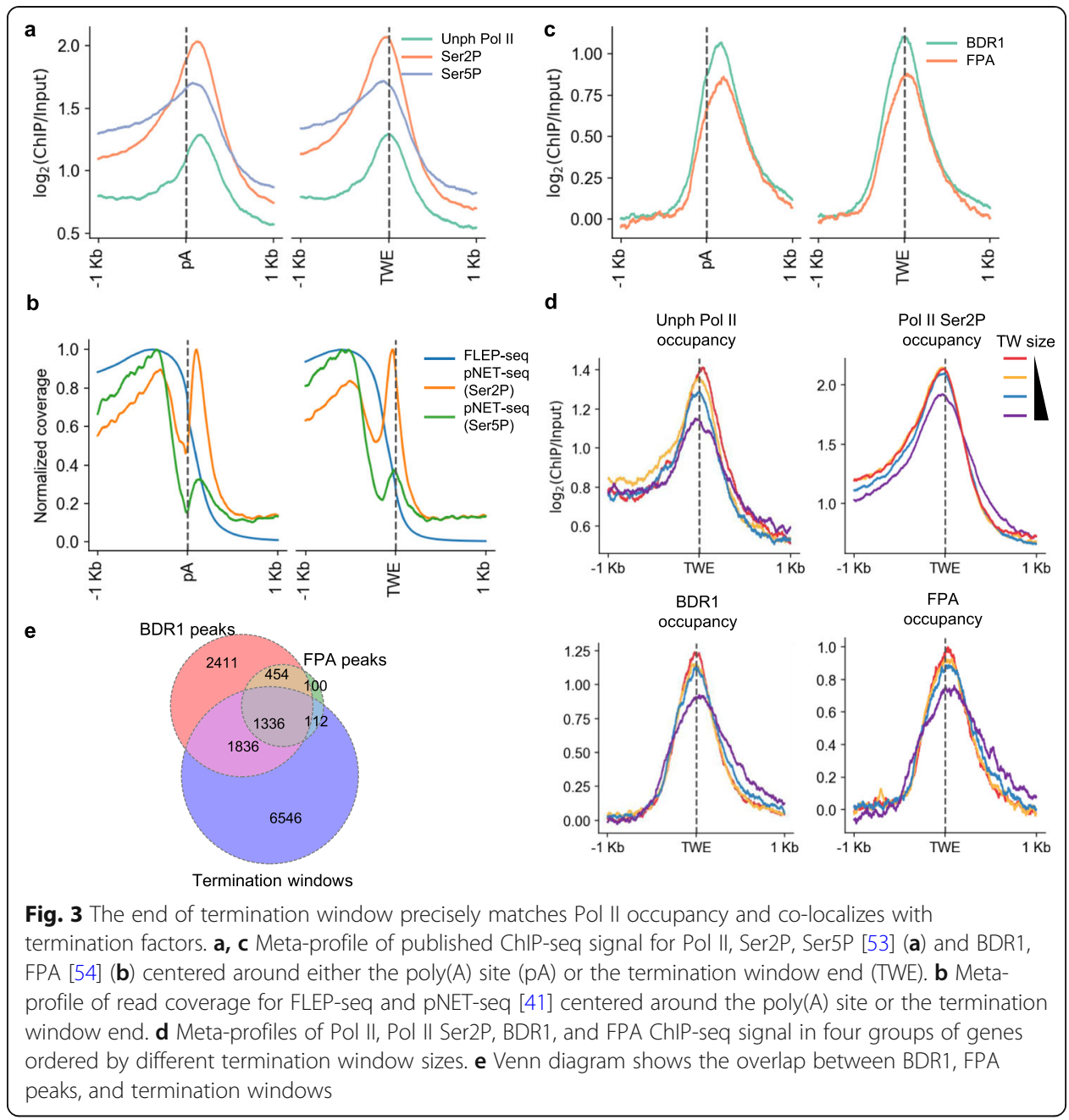

During the termination process, many factors are associated with the Pol II elongation complex [1]. We found that BDR1, a negative elongation factor that prevents transcriptional interference in Arabidopsis [54], localized precisely at the end of the termination window (Fig. 3c). Similarly, an mRNA 3 '-end processing factor FPA that controls the cleavage and polyadenylation $[12,55,56]$ is found to coincide with BDR1 distribution (Fig. 3c). In addition, ATAC-seq data [57] showed a preference for open chromatin status at the end of termination window (Additional file 1, Fig. S3b), consistent with the previous report that nucleosome depletion is linked to the 3 '-end formation [58]. While poly(A) sites are featured with low CG methylation and low GC content compared to flanking regions, we did not observe obvious change of DNA methylation or GC contents at major site for transcription termination (TWE) (Additional file 1, Fig. S3c, d). We also found that the strength of Pol II occupancy is negatively correlated with TW size (Fig. 3d), and similar trends were observed in the distribution of BDR1 and FPA. Previous study demonstrated that BDR proteins prefer to reside at gene borders, in both transcription start sites (TSS) and transcription end site (TES) [54]. It is worth to clarify that poly(A) site, TES, and TWE are different from each other-poly(A) site is where cleavage and polyadenylation occurs; TES in Araport11 is defined based on the reconstructed transcript assembly using a collection of 
Illumina RNA-seq [19], and TWE is the median readthrough length defined by FLEPseq data (Additional file 1, Fig. S1d). Our results further revealed that most of FPA peaks overlapped with BDR1 peaks at termination window (Fig. 3e). Nevertheless, only a small portion of termination windows contained BDR1 and FPA peaks at their ends (Fig. 3e), suggesting many other factors are potentially involved in the termination process in Arabidopsis.

\section{tRNA genes promote efficient termination of Pol II transcription}

The tRNA genes are transcribed by Pol III [59]. In this study, we discovered a termination mechanism for Pol II that is shaped on the downstream tRNA genes. In the compact Arabidopsis genome, we identified in total more than 60 Pol II genes that have adjacent tRNA genes immediately downstream (distance to downstream gene $<200 \mathrm{nt}$ ) (Additional file 3, Table S2). In the tandemly arrange case, the readthrough transcripts terminated sharply at $60 \mathrm{nt}$ upstream of the tRNA gene, and the distance between termination window end and downstream tRNA is consistently at $\sim 60 \mathrm{nt}$ (Fig. 4a, b). Our results showed that this highly efficient pattern of termination is specific to genes to which a tRNA gene locates downstream (Fig. 4c; Additional file 1, Fig S4). For the 34 genes whose termination is regulated by the downstream tRNA in tandem direction, 26 of them have termination window ends located $\sim 60 \mathrm{nt}$ upstream of the tRNAs. While in the convergently arrange cases, the distance between the termination window end and tRNA is around $10 \mathrm{nt}$ with a less obvious concentration (Fig. 4d, e), and these genes also terminated more efficiently than those with non-tRNA immediately downstream (Fig. 4f). Previous report estimated that RNA polymerase may contact up to 90 bp of DNA $(-70$ to +20$)$ at promoter regions [60]. Moreover, previous literature has shown that Pol II pausing or stalling can be influenced by chromatin structure [2]. Therefore, it is possible that the termination of Pol II elongation at - $60 \mathrm{nt}$ is caused by a unique chromatin status at the tRNA gene promoter. To test this scenario, we analyzed published data of MNase-seq [61], ChIP-seq (H3K4me3, H3K36me3, H3K27me3, H3K9me2) [62], and the CG, CHG, and CHH DNA methylation level to check the chromatin status at the two tRNA loci showed in Fig. 4, including nucleosome positioning, histone modification, and DNA methylation (Additional file 1, Fig. S5). We did not observe an obvious enrichment or depletion of the common epigenetic marks around the tRNA promoters. Future studies on the factors that bind to the promoter of tRNA gene may help to explain its role in blocking Pol II transcription. Besides Arabidopsis, a previous report in Leishmania major also found tRNA gene can serve as terminator for Pol II transcription in the trypanosomatida [63].

We also analyzed the correlation between termination window size and intergenic distance, direction, and transcription level of downstream protein-coding and/or noncoding genes, respectively. The genome-wide analysis showed that the termination window size of genes arranged in tandem is similar to genes arranged in convergent direction (Additional file 1, Fig. S6a). We found that termination window size is slightly positively correlated with intergenic length and slightly negatively correlated with the transcription level of downstream genes (Additional file 1, Fig. S6b-d). We proposed that the pairing between Pol II gene and their downstream tRNA may be evolutionarily beneficial for assisting efficient termination in a small and compact genome (Fig. $4 \mathrm{~g}$ ). 


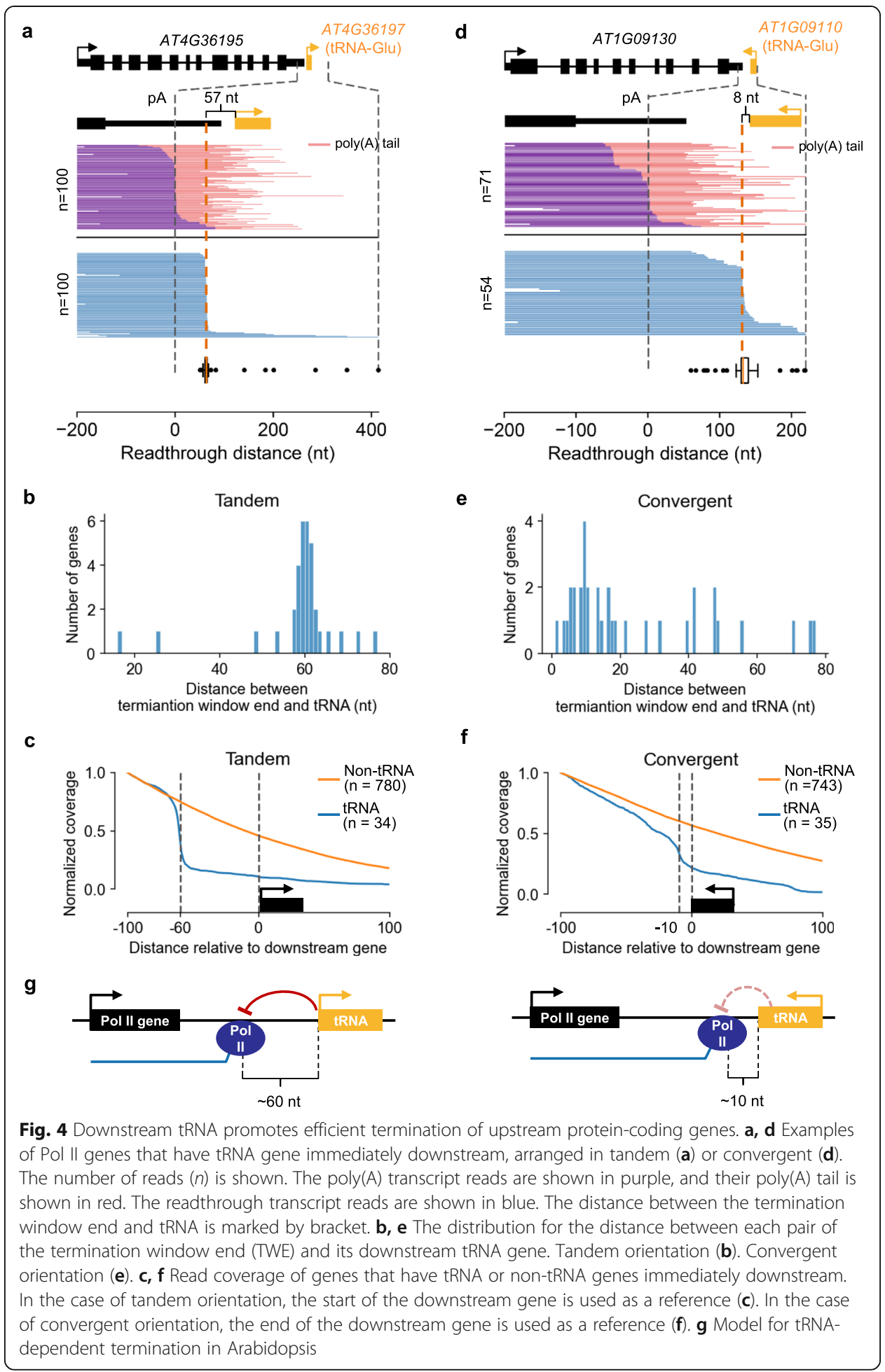

\section{Mutation of AtXRN3 delays the termination of cleaved readthrough transcription}

Many previous studies have supported the torpedo model for transcription termination, in which a nuclear $5^{\prime} \rightarrow 3^{\prime}$ exonuclease plays the central role in degrading the $3^{\prime}$ cleavage products, and eventually catch up with elongating Pol II to expel it from DNA template via kinetic competition [2, 16, 18]. In Arabidopsis, two nuclear exonucleases 
AtXRN2 and AtXRN3 are orthologs of the human Xrn2 [11, 48]. Although they share extensive sequence similarities, only AtXRN3 is shown to be the primary exonuclease involved in Pol II termination [14, 47, 64]. RNA-seq analysis performed with total RNAs from weak alleles of atxrn3 show increased signal of mostly polyA+ mRNAs downstream of poly(A) site compared to wildtype [14, 47]. However, it remains unclear if the 3' cleavage products without poly(A) tails accumulated in atxrn3 and how atxrn3 affects readthrough, due to the limitation of short-read sequencing. We set out to address these questions by taking advantage of our full-length nascent RNA sequencing method, which can capture the 3 ' cleavage products, particularly those without poly(A) tails, enriched in the partial loss-of-function allele of atxrn3. In order to maximize the capture rate of 3' cleavage products which might not be closely associated with chromatin, we used nuclear RNA instead of chromatin-bound RNA as the nascent RNA input of FLEP-seq. It turned out that both the nuclear fraction and the chromatin-bound fraction can efficiently capture the cleaved and readthrough transcripts (Additional file 1, Fig. S7a). Since nuclear RNA isolation involves fewer steps than chromatin-bound RNA isolation, we proceeded with nuclear RNA to make FLEP-seq libraries in a series of mutants and wildtype controls (please see "Methods" for detail). The termination window sizes from wildtypes using chromatin-bound RNA and nuclear RNA are highly consistent (Additional file 1, Fig. S7b), suggesting that nuclear RNA is a viable substitute for chromatin-bound RNA in studying transcription termination in Arabidopsis.

Compared to wildtype, the 3 ' cleavage products drastically accumulated in the atxrn 3 mutant (Fig. 5b), consistent with the function of AtXRN3 in $5^{\prime} \rightarrow 3^{\prime}$ degradation of co-transcriptional cleavage products $[9,11,14]$. This accumulation leads to a clear peak of the $5^{\prime}$ end of cleaved readthrough reads (3' cleavage products) at poly(A) site in atxrn3 mutant, which is absent in the wildtype control library (Fig. 5a). Similar results were also observed in human Xrn2 depletion cell line detected by short-read-based method POINT-5 [25]. In addition, accumulation of the 3' cleavage products is not influenced by splicing, as genes with or without splicing have 3' cleavage products enriched in atxrn3 and aligned accurately at the poly(A) site (Fig. 5b, Fig. S8). From a genome-wide perspective, we compared the size of termination windows in the atxrn 3 mutant and in wildtype, and the result showed a strong impact of atxrn 3 on termination window size at hundreds of gene loci (Fig. 5d). The termination window of 354 genes were statistically longer in the atxrn 3 mutant than in wildtype (Mann-Whitney $U$ test, $p$ value $<0.001$ ). These results illustrated that AtXRN3 is specifically responsible for the degradation of $3^{\prime}$ cleavage products in vivo.

In addition to atxrn3, we also characterized the fpa and met1 mutants by FLEP-seq. FPA is a component of the $3^{\prime}$ end processing complex, and MET1 is the key DNA methyltransferase in Arabidopsis [65]. The fpa mutant FLEP-seq data showed a global impact on termination window size and a prolonged 3 ' end distribution compared to WT (Fig. 5c, d), consistent with its previously reported function as a termination factor $[12,56]$. We also found some interesting cases that support the previous report of chimeric transcripts and cryptic splicing occurring in the fpa mutant $[12,56]$. For example, some readthrough transcripts in the fpa mutant can extend into downstream genes to form chimeric RNAs accompanied by the cryptic splicing event (Additional file 1, Fig. S9a). Cryptic splicing event in the fpa mutant can even result in excision of the entire gene in the middle from the readthrough transcript that spans multiple genes 


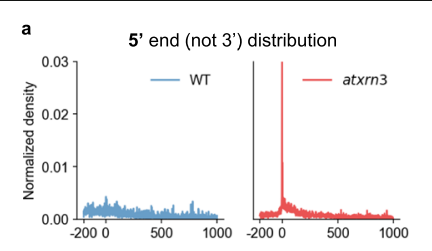

b
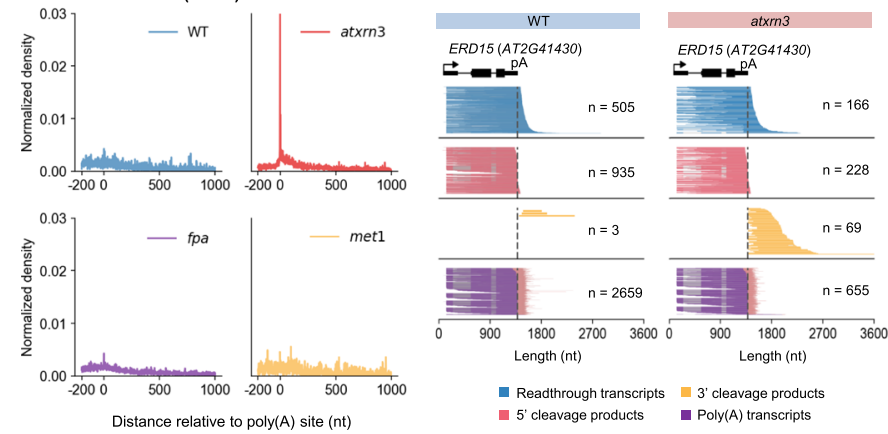

c

3' end distribution
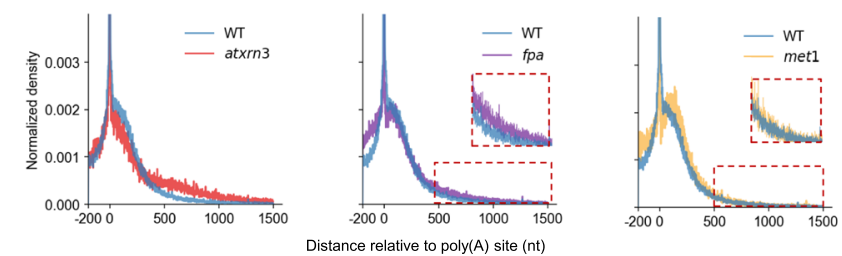

d

Termination window size (nt)

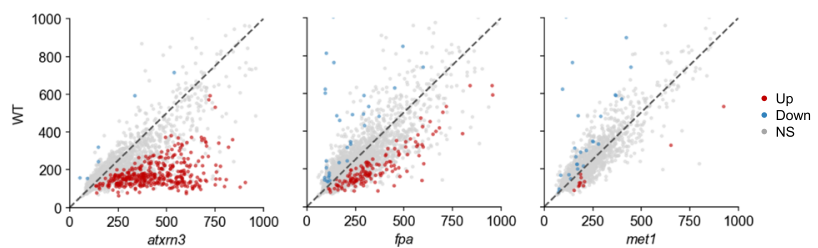

Fig. 5 3' cleavage products accumulate in the atxrn3 mutant. a 5' end distribution for the non-poly(A) reads in wildtype atxrn3, fpa, and met1 mutant near poly(A) site. b Examples of genes with accumulated 3' cleavage products in atxrn3 mutant. Left panel, wildtype; right panel, atxrn3 mutant. c Comparison of 3' end distribution for the non-poly(A) reads in wildtype and atxrn3, fpa, met 1 mutant near poly(A) site (the maximum limit of the $y$-axes is set at 0.003 to highlight differences at low-signal region). The zoomed-in view is shown in red dashed box. $\mathbf{d}$ Comparison of termination window size per gene between wildtype and mutants. The $p$ value was calculated using a Mann-Whitney $U$ test. NS, not significant $(p>0.001)$

(Additional file 1, Fig. S9b). In the met1-1 mutant of DNA methyltransferase MET1 [65], in which most CG methylation at genic regions is lost (Additional file 1, Fig. S13a), the distribution for 3' ends of readthrough RNAs is largely unaffected (Fig. 5c), and the size of termination windows is similar to that in wildtype Col-0 (Fig. 5d). However, it is worth noting that met1-1 is not a null allele and still have some remaining CG methylation at TE regions (Additional file 1, Fig. S13b), recent characterization using ONT direct RNA sequencing of full-length mRNA from the strong met1-3 allele, which removes virtually all CG methylation, has discovered the effects of DNA methylation on splicing site and poly(A) site selection, as well as on poly(A) tail length [66].

Furthermore, we identified 14 genes with cleaved readthrough transcripts entering their immediate downstream genes in atxrn3 mutant (Additional file 4, Table S3). For example, AT1G73510 is a pollen-specific gene that is not expressed in seedlings (Additional file 1, Fig. S10), the materials used in our FLEP-seq libraries. In atxrn3, readthrough from its upstream gene NUDT21 (AT1G73540) and ORRM6 (AT1G73530) can continue elongation and pass through the entire downstream gene, and then be cleaved and polyadenylated (Fig. 6a). Strikingly, our full-length data revealed that the 
readthroughs in $\operatorname{arxtn} 3$ can be cleaved and polyadenylated multiple times as they elongate through several subsequent poly(A) sites in a row (Fig. 6a, magnified view). In addition, we found that cleaved readthrough transcription can yield normally spliced and polyadenylated mRNAs without their own transcription initiation (Fig. 6a). This can be clearly seen with a zoomed-in view around the TSS site of AT1G73530 in two biological replicates of the wildtype and atxrn3 FLEP-seq libraries, showing that some polyadenylated transcripts of AT1G73530 in atxrn3 originated from the upstream poly(A) site as cleavage products of upstream transcriptional readthrough, instead of their own initiation (Fig. 6b). A reordered view of reads at AT1G76180-AT1G76170 region also confirms this observation, with most reads of the downstream AT1G76170 come from the cleaved readthrough of the upstream gene AT1G76180 (Additional file 1, Fig. S11). Besides nascent RNA, we also check the mRNA level by analyzing previously published RNA-seq data of wide-type and atxrn3 mutant [14]. The coverage plot confirmed that more reads are aligned to the intergenic region of AT1G73540AT1G73530 in atxrn3 mutant, compared to the fewer reads at the same regions in wildtype (Fig. 6c). It remains unclear if these mRNAs originated from 3' cleavage products can be translated, as they may lack the 5' cap structure. Previous work on AtXRN3 proposed several models to explain the elevated poly(A) + RNA-seq signal downstream of poly(A) site, including a role for transcription activation of downstream genes by readthrough transcription [14, 47]. Our single-molecule nascent RNA data suggests that readthrough transcription itself could be enough to drive the production of multiple downstream transcripts, highlighting the importance of AtXRN3-mediated transcription termination in the compact Arabidopsis genome (Fig. 6d).

However, the AT1G73530-AT1G73510 fusion poly(A) transcripts cannot be solely explained by the loss of $5^{\prime}$ to $3^{\prime}$ exonuclease activity as AtXRN3 should not affect the cleavage at the poly(A) site. It is possible that AtXRN3 may affect chromatin status that in turn determines poly(A) site selection and the readthrough phenomena. To check if DNA methylation status is altered at poly(A) sites in the atxrn3 mutant, we performed whole-genome bisulfite sequencing (WGBS) in seedlings of atxrn3 and corresponding Col-0 control. At the AT1G73530-AT1G73510 loci, there is little CG methylation in the wildtype, and it remains mostly unmethylated in the atxrn3 mutant (Additional file 1, Fig. S12a). This is consistent with previous DNA methylation profiling of multiple wildtype Arabidopsis libraries from the Jacobsen group [67] (Additional file 1, Fig. $\mathrm{S} 12 \mathrm{~b})$. In addition, we found that the overall DNA methylation pattern at genic regions remains unchanged in the atxrn3 mutant compared to WT in CG, CHG, and $\mathrm{CHH}$ contexts (Additional file 1, Fig. S13c). However, individual poly(A) sites could still be affected, as a recent work has discovered large number of novel TTSs in the met1-3 mutant, suggesting a role of DNA methylation in poly(A) site selection [66]. We proposed that, besides exonuclease activity of AtXRN3, other factors such as chromatin status and DNA modification could also contribute to the accumulated readthrough transcripts in the atxrn3 mutant.

\section{Discussion}

Here we applied single-molecule Nascent RNA sequencing to investigate the transcription termination landscape at a genome-wide level in Arabidopsis. The single-molecule feature of Nanopore sequencing enables our FLEP-seq to analyze the termination 


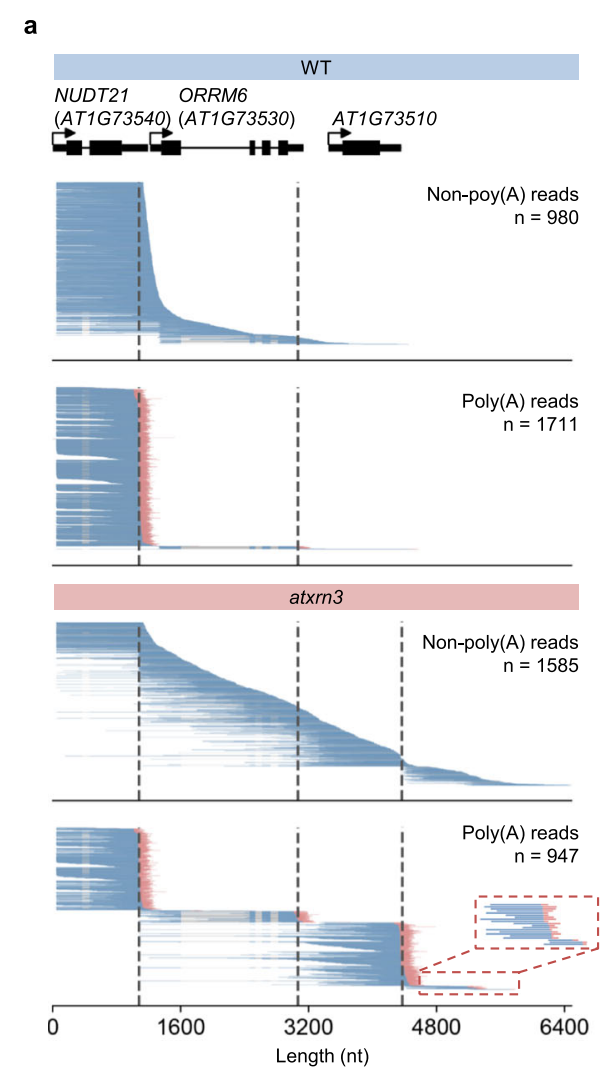

c

RNA-seq coverage (Krzyszton et al., 2018)

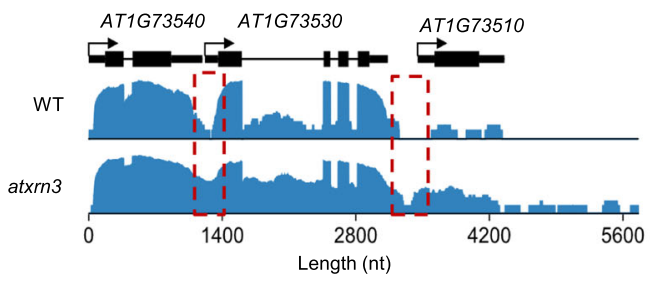

b

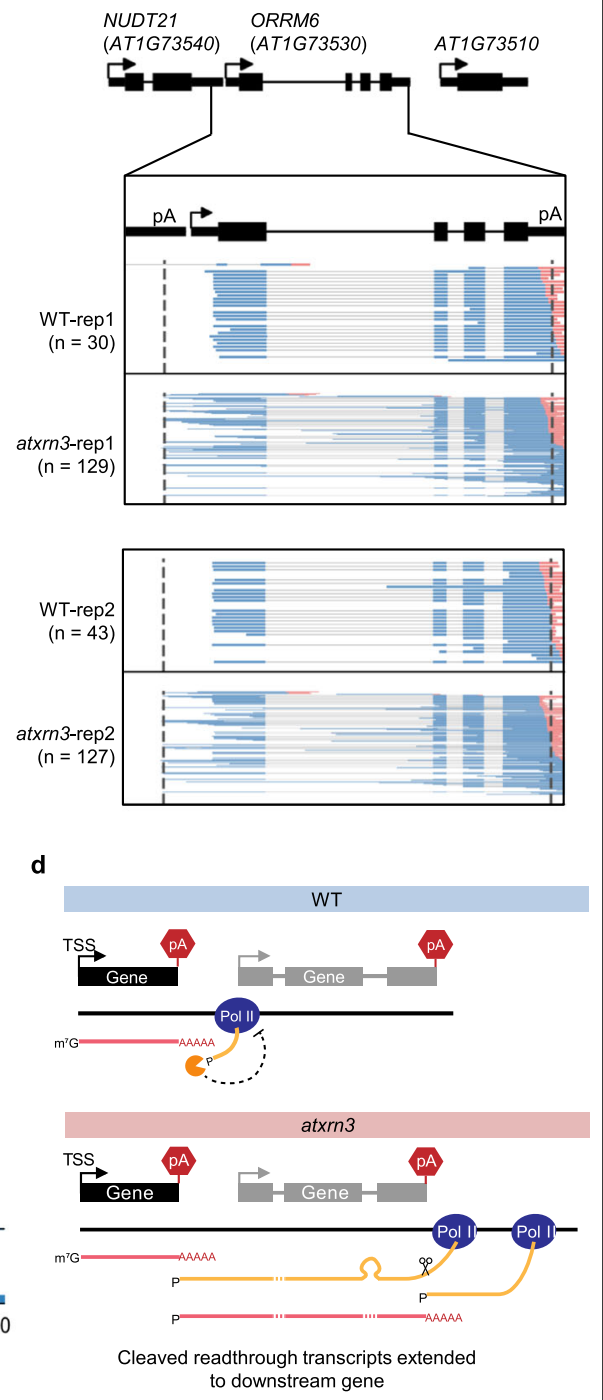

Fig. 6 Cleaved readthrough transcripts can transcribe into downstream genes and produce spliced and polyadenylated mRNAs in atxrn3. a Examples of genes with cleaved readthrough reads extended to downstream genes. Upper panel, wildtype; lower panel, atxrn3 mutant. The black dashed line indicates the poly(A) site. The red dashed box showed the magnified view of cleaved and polyadenylated reads at the subsequent poly(A) site. $\mathbf{b}$ Zoomed-in view of poly(A) reads aligned to gene AT1G73530 in two biological replicates of the wildtype and atxrn3 FLEP-seq libraries. c Read coverage of RNA-seq data [14] around the AT1G73540-AT1G73510 region in wildtype and atxrn3 mutant ( $\log _{2}$ scale). d Model of AtXRN3-mediated transcription termination

window of genes (Fig. 2e), regardless of how narrow the intergenic regions are (Fig. 1e). In addition, our data allows the precise and robust identification of poly(A) sites in the same FLEP-seq library (Fig. 1b; Additional file 1, Fig. S1), which can facilitate the accurate analysis of PAS-dependent termination. Besides, in stress or mutant conditions, 3'end cleavage may occur at novel poly(A) sites [43]. Therefore, FLEP-seq is suitable to study PAS-dependent termination under different conditions and genetic backgrounds.

Many powerful Illumina-based methods have been applied to characterizing transcription termination, including GRO-seq, NET-seq, 4tU-seq, and TT-seq [15, 17, 21, 24]. Several protocols also take advantages of the paired-end sequencing function of 
Illumina to distinguish the $5^{\prime}$ and $3^{\prime}$ ends of each RNA molecule, such as TIF-seq [68]. The Illumina platform also holds the advantage of lower cost therefore easier to achieve deeper sequencing yields. Nevertheless, our Nanopore-based single-molecule approach has several unique advantages compared to previous Illumina-based methods-(a) There is no RNA fragmentation step involved in the FLEP-seq procedure; therefore, it can capture the full-length RNA intermediates during termination, with information on both their $5^{\prime}$ end and $3^{\prime}$ end positions, as well as the poly(A) tail; (b) FLEP-seq can separate the cleaved and uncleaved readthrough RNAs that are previously indistinguishable in the short-read sequencing datasets; (c) The single-molecule measurement enables us to quantify the termination window using median readthrough distance, which is less vulnerable to fluctuations in sequencing depth compared to coveragebased assessment from previous Illumina-based methods. Hence, with these unique features, FLEP-seq could serve as a new method for analyzing transcription termination that is complementary to previous Illumina-based methods. Besides, metaboliclabeling-based methods, such as $4 \mathrm{t} U$-seq and $4 \mathrm{sU}$-seq, can detect newly synthesized RNA and can be combined with long-read sequencing platforms to characterize fulllength nascent RNA [34].

Pol II pausing after poly(A) site is a common feature in plant transcription [39, 41]. Previous studies have proposed that Pol II pausing downstream of poly(A) site promotes the mRNA 3 '-end processing and subsequent torpedo degradation for termination $[18,69]$. Consistent with this idea, we observed that the 3 '-end processing factor FPA and negative elongation factor BDR1 co-localize with Pol II at a set of genes (Fig. 3). Future application of FLEP-seq to study termination changes in the mutants of the termination factors, such as BDR1 and CPSF components, could further expand our understanding of the termination mechanism in plants. Another important question is what are the potential determinants that make hundreds of genes sensitive to atxrn3 mutation. We do not have a clear answer yet, but it is worth noting that the atxrn3 alleles we and other previous studies sequenced are weak alleles, and the strong allele of atxrn3 is embryo lethal [48], which suggests AtXRN3 may have a much stronger impact than revealed from the weak alleles.

FLEP-seq approach has its limitations [38]. For example, when RNA is reverse transcribed into cDNA, the integrity of long reads relies on the success of a complete reverse transcription, which favors shorter RNAs. This problem is particularly relevant for animal nascent RNAs with long introns, and recent advancements in Nanopore direct RNA sequencing technology may help to improve this shortcoming [26, 30, 33, 34, 56]. However, the current generation of ONT DRS technology has several issues that limit its use in characterizing transcription termination: (a) The current ONT DRS kit is designed for profiling polyA+ RNAs, additional steps such as in vitro polyadenylation [33, 34], or using customer-specific RT adaptor [70, 71], can be applied to characterize non-polyA RNAs; (b) DRS has much lower yields per run $(\sim 1$ million reads per flow cell on MinION) compared to cDNA sequencing $(\sim 10$ million reads per flow cell on MinION); (c) DRS requires much higher input (500 to $1000 \mathrm{ng}$ ) compared to the as little as $1 \mathrm{ng}$ needed for FLEP-seq. We hope that the future generation of ONT DRS will be able to address these issues so that it can be more easily applied on non-polyA RNAs. 


\section{Conclusions}

Our single-molecule full-length nascent RNA sequencing approach presented here has the unique advantage of distinguishing the full-length readthrough, cleaved, and polyadenylated forms of transient RNA intermediates in the termination process and enables the characterization of transcription termination patterns at the single-gene level in the model plant Arabidopsis.

\section{Methods}

Plant materials and growth conditions

Arabidopsis thaliana wildtype (accession Columbia-0) and T-DNA insertion line of atxrn3 (SALK_116909C), fpa (SALK_011615), and met1-1 [65] were used in this study. Seeds were grown on $1 / 2 \mathrm{MS}$ medium at $22^{\circ} \mathrm{C}$ ( $16 \mathrm{~h}$ light $-8 \mathrm{~h}$ dark) for 12 days before collection. Twelve-day-old seedlings were harvested and immediately frozen in liquid nitrogen, then stored at $-80^{\circ} \mathrm{C}$ for RNA extraction.

\section{Nuclear RNA extraction and FLEP-seq library construction}

Nuclear RNA extraction is performed according to the previously reported method [37, 38]. In brief, $2 \mathrm{~g}$ of seedlings was ground with liquid nitrogen and transferred to an icecold RNase/DNase-free 50-ml tube with $10 \mathrm{ml}$ Honda buffer (0.44 M sucrose, 1.25\% (w/v) Ficoll, 2.5\% (w/v) dextran T40, $20 \mathrm{mM}$ HEPES-KOH pH 7.4, $10 \mathrm{mM} \mathrm{MgCl}_{2}, 0.5 \%$ (w/v) Triton X-100, $1 \mathrm{mM}$ dithiothreitol (DTT), 1× protease inhibitor (Roche), and 100 $n g \mathrm{l}^{-1}$ tRNA). The samples were homogenized and filtered through Miracloth, and the remaining samples on filter were washed with another $10 \mathrm{ml}$ Honda buffer. After centrifuged at $2000 \mathrm{~g}$ for $5 \mathrm{~min}$ at $4{ }^{\circ} \mathrm{C}$, the nuclear pellets were resuspended and washed twice with $15 \mathrm{ml}$ Honda buffer. The pellets were transferred to a 1.5-ml RNase/DNasefree microcentrifuge tube in $1 \mathrm{ml}$ Honda buffer and centrifuged at $8000 \mathrm{~g}$ for $1 \mathrm{~min}$ at $4{ }^{\circ} \mathrm{C}$. Then the supernatant was completely removed. For RNA extraction, the nuclei pellet was resuspended with $1 \mathrm{ml}$ TRIzol and vortexed to mix thoroughly. After $10 \mathrm{~min}$ incubation at room temperature, $0.2 \times$ volume of chloroform was added, mixed, and incubated at room temperature for $5 \mathrm{~min}$. The mixture was centrifuged at $14,000 \mathrm{~g}$ for 10 min at $4{ }^{\circ} \mathrm{C}$. The supernatant was collected and added with one volume of $100 \%(\mathrm{w} / \mathrm{v})$ ethanol. The RNA extraction was performed according to the manufacturer's instructions (ZYMO, R2070). RNA concentration was measured by Nanodrop and Qubit 3.0 fluorometer with Equalbit RNA HS assay kit (Vazyme, EQ211-01).

At least $2 \mu \mathrm{g}$ nuclear RNA was used for FLEP-seq library preparation. Ribosomal RNA (rRNA) was depleted using riboPOOL kit (siTOOLs Biotech) following the manufacturer's instructions. After purification with the ZYMO RNA Clean \& Concentrator-5 kit (ZYMO, R1013), the nuclear RNA was mixed with 50 pmol 3' adapter (5'rAppCTGTAGGCACCATCAAT-NH2-3', NEB, S1315S) at $65^{\circ} \mathrm{C}$ for $5 \mathrm{~min}$, and then placed on ice for more than $1 \mathrm{~min}$. Then, $2 \mu \mathrm{l} 10 \times \mathrm{T} 4 \mathrm{RNA}$ ligase reaction buffer (NEB, M0242), $10 \mu \mathrm{l}$ 50\% PEG 8000 (NEB, M0242), $1 \mu \mathrm{l} 40 \mathrm{U}_{\mu} \mathrm{l}^{-1}$ Murine Rnase Inhibitor (Vazyme, R301-03), and $1 \mu \mathrm{T} 4$ RNA ligase 2, truncated K227Q (NEB, M0242) were added to the RNA and mixed thoroughly. The ligation reaction was performed at $16{ }^{\circ} \mathrm{C}$ for $10 \mathrm{~h}$. After that, the RNA was purified and concentrated to $6 \mu \mathrm{l}$ using the ZYMO RNA Clean \& Concentrator kit (ZYMO, R1013). Reverse transcription and template- 
switching were performed using the SMARTer PCR cDNA Synthesis Kit (Takara, 634926) with minor modification. The SMART CDS Primer II A was replaced by the custom 3' cDNA RT primer (5' -AAGCAGTGGTATCAACGCAGAGTACATTGATG GTGCCTACAG-3'), which is complementary to the $3^{\prime}$ adapter sequence. To minimize the PCR bias resulting from over-amplification, PCR cycle optimization was performed to determine the best cycle number for cDNA amplification. The cDNA for Nanopore library construction was amplified using the optimized cycle number and purified twice with the VAHTS DNA Clean Beads (Vazyme, N411). DNA concentration and quality were measured by Qubit 3.0 and Agilent 2100 Bioanalyzer. Then, 200 fmol DNA was used to construct the Nanopore library using the Ligation Sequencing Kit (SQKLSK109, Oxford Nanopore Technologies) according to the instruction. Libraries were loaded onto R9.4 flow cell (Oxford Nanopore Technologies) and sequenced on MinION device for $\sim 48 \mathrm{~h}$.

\section{Nanopore data processing}

The Nanopore data analysis workflow is provided in Additional file 1, Fig. S2. The Nanopore data pre-processing was performed as previously described with minor modification [37, 38]. Guppy basecaller (Oxford Nanopore Technologies, v4.0.11) was used to convert Nanopore raw signal to sequence, with parameters --c dna_r9.4.1 450bps_hac.cfg, --qscore_filtering. The basecalled reads were mapped to Arabidopsis TAIR10 genome using Minimap2 v2.10-r761 [72] with parameters: -ax splice, -secondary $=n o,-G 12000$. The unmapped reads, not primary alignment reads, or supplementary alignment reads were removed using SAMtools [73] view with parameter - F 2308. The $5^{\prime}$ or $3^{\prime}$ unmapped sequences (soft-clip sequences) plus the flanking $20 \mathrm{nt}$ mapped sequences were used to search the template-switching oligo sequence (5' -AAGCAGTG GTATCAACGCAGAGTACATGGG-3') and the 3' adapter sequence (5'-ATTG ATGGTGCCTACAG-3') using our custom script adapterFinder.py. The templateswitching oligo and the $3^{\prime}$ adapter can characterize the integrity of $5^{\prime}$ and $3^{\prime}$ end of reads and provide the strand information. Therefore, only reads containing both sequences were used for subsequent analysis. PolyAcaller was used to estimate poly(A) tail lengths of Nanopore reads [38].

\section{Identification of poly(A) sites}

The identification of poly(A) sites was performed as previously described [45]. Briefly, the 3' end of poly(A) transcript read was considered as the poly(A) site. Due to the heterogeneity of poly(A) sites within the same gene in Arabidopsis, the poly(A) sites within $24 \mathrm{nt}$ of each other within the same gene were grouped into a poly(A) site cluster (PAC). The PAC with less than three poly(A) sites was discarded. The poly(A) site with the greatest number in the PAC was defined as the representative poly(A) site. The representative poly(A) sites were used in the subsequent analysis. To eliminate the influence of alternative polyadenylation, only genes with a single representative poly(A) site were used for further analysis.

\section{Classification of RNA intermediates produced during termination}

The schematic of RNA intermediates is shown in Fig. 1a. In detail, reads are categorized as "poly(A) transcripts" if their poly(A) tail lengths are greater than $15 \mathrm{nt}$ as 
previously described $[37,38]$. To eliminate the influence of sequencing error on the 3 ' end accuracy, the remaining reads are considered as non-poly(A) reads if the distance between the 3' adapter and the mapping region is less than $5 \mathrm{nt}$. Pysam [73] (https:// github.com/pysam-developers/pysam) was used to extract the $5^{\prime}$ and $3^{\prime}$ end coordinates of reads from BAM files. Non-poly(A) reads with $5^{\prime}$ ends located at gene body ( $>50 \mathrm{nt}$ upstream of poly(A) site) and $3^{\prime}$ end located more than $50 \mathrm{nt}$ downstream of the poly(A) site are considered as "readthrough transcripts." Non-poly(A) reads with 5' ends located at gene body as well as $3^{\prime}$ ' ends within 50 nt upstream and downstream of poly(A) sites are considered as " 5 ' cleavage products." Non-poly(A) reads with 5 ' end within $500 \mathrm{nt}$ downstream of poly(A) sites are regarded as " 3 ' cleavage products." Of note, when two neighboring genes are arranged closely on the same strand, the " 3 ' cleavage products" is considered only if the $5^{\prime}$ end is $100 \mathrm{nt}$ before the TSS of the downstream gene.

\section{Calculation of termination window size}

The readthrough distance was defined as the distance between poly(A) site and 3' end of each read (Fig. 2a). Genes with a minimum read count threshold of 15 (readthrough transcript reads +3 ' cleavage product reads) were used. For each gene of interest, we use the median readthrough distance to represent the termination window size, to reduce the effect of sequencing depth (Fig. 2b-d).

\section{Whole-genome bisulfite sequencing and analysis}

Genomic DNA was extracted from 12-day-old seedlings of wildtype and xrn3 mutant using Hi-DNAsecure Plant Kit (TIANGEN, Cat. DP350-02). The whole-genome bisulfite sequencing (WGBS) libraries were prepared and sequenced at the BGI Group on the MGISEQ-2000RS. For each library, adapters were trimmed by using fastp v0.20 [74], and reads were mapped to Arabidopsis TAIR10 genome using BSMAP [75]. The DNA methylation level in each $100 \mathrm{bp}$ bin was calculated as the ratio of methylated cytosines to the total number of cytosines $(\# \mathrm{C} /(\# \mathrm{C}+\# \mathrm{~T}))$ [67].

\section{Illumina data processing}

Illumina reads were first trimmed the adapter sequences using fastp v0.20 [74]. For the pNET-seq, plaNET-seq, and GRO-seq data, the reads were mapped to the Arabidopsis TAIR10 genome using STAR v2.7.2b [76]. Only the uniquely mapped reads with mapping quality greater than 10 were used for further analysis. For ChIP-seq and ATACseq data, reads were mapped to Arabidopsis TAIR10 genome using Bowtie2 v2.3.5.1 [77], with parameters -dovetail, and with parameters $-X 1000$ for paired-end sequencing data to set the maximum insert size. The duplicate reads were removed by using Picard v2.2.4 in MarkDuplicates mode (http://broadinstitute.github.io/picard/). For ChIP-seq data, input-normalized $\log _{2}$ fold enrichment was computed using deepTools bamCompare [78], with parameters --binSize 10 --scaleFactorsMethod SES. ChIP-seq peaks were called on input and control BAM files with MAC2 [79]. Normalized coverage was computed using deepTools bamCoverage [78], with parameters --binSize 10 --normalizeUsing RPGC --effectiveGenomeSize 119481543. The normalized coverage was directly used to produce meta-profile without smoothing. 


\section{Quantification and statistical analysis}

Pearson's correlation coefficient is used to measures the statistical relationship. MannWhitney $U$ test was used to obtain the significance between conditions. All information about statistical testing can be found in figure legends. This includes the number of observations, statistical tests used, and significance level.

\section{Supplementary Information}

The online version contains supplementary material available at https://doi.org/10.1186/s13059-021-02543-4.

Additional file 1: Figures S1-13. Supplementary figures

Additional file 2: Table S1.The termination window size and poly(A) site identified by FLEP-seq data.

Additional file 3: Table S2. Closely spaced genes with tRNA located immediately downstream.

Additional file 4: Table S3. Genes with cleaved readthrough transcripts enter the downstream genes in atxrn3.

Additional file 5. Review history.

\section{Review history}

The review history is available as Additional file 5.

Peer review information

Wenjing She was the primary editor of this article and managed its editorial process and peer review in collaboration with the rest of the editorial team.

\section{Authors' contributions}

W.M., B.L., X.J., D.L., Y.Y., J.J., and Y.Long performed the experiments. W.M., B.L., H.Z., Y.Y., J.J., and J.Z. analyzed the data X.D., X.C., Y.Liu, and H.G. provided materials. J.Z. conceived and oversaw the study. W.M. and J.Z. wrote the draft and all authors revised the manuscript. All author(s) read and approved the final manuscript.

\section{Funding}

The group of J.Z. is supported by the National Key R\&D Program of China Grant (2019YFA0903903); the Program for Guangdong Introducing Innovative and Entrepreneurial Teams (2016ZT06S172); the Shenzhen Sci-Tech Fund (KYTD PT20181011104005); the Key Laboratory of Molecular Design for Plant Cell Factory of Guangdong Higher Education Institutes (2019KSYS006); and the Stable Support Plan Program of Shenzhen Natural Science Fund Grant (20200925153345004).

\section{Availability of data and materials}

The nuclear RNA FLEP-seq and WGBS data generated in this paper have been deposited in the Genome Sequence Archive in National Genomics Data Center, China National Center for Bioinformation / Beijing Institute of Genomics, Chinese Academy of Sciences, under accession number CRA005016 and CRA005017 [80]. The chromatin-bound RNA FLEP-seq data have been deposited in NCBI's Sequence Read Archive (SRA): PRJNA591665 [37, 38]. The FLEP-seq data are also displayed in a JBrowse (https://zhailab-sustech.github.io/jbrowse2/). All other relevant data supporting the key findings of this study are listed as follows. GRO-seq data were acquired from published studies (GEO accession: GSE83108, GSE100010, and GSE109974) [39-41]. pNET-seq and plaNET-seq data were obtained from published studies (GEO accession: GSE109974 and GSE131733) [41, 42]. Pol II, Ser2P, FPA, and BDR1 ChIP-seq data were obtained from published studies (GEO accession: GSE95301 and GSE112443) [53, 54]. ATAC-seq data were obtained from the published studies (GEO accession: GSE85203) [57].

The pipeline developed for pre-processing FLEP-seq data is available at https://github.com/ZhaiLab-SUSTech/FLEPSeq. All scripts used for transcription termination analysis in this study are available at Github (https://github.com/ZhaiLabSUSTech/Termination_landscape) [81] or Zenodo (https://doi.org/10.5281/zenodo.5607518) [82].

\section{Declarations}

Ethics approval and consent to participate

Not applicable.

\section{Competing interests}

The authors declare that they have no competing interests.

\section{Author details}

${ }^{1}$ Department of Biology, School of Life Sciences, Southern University of Science and Technology, Shenzhen 518055, China. ${ }^{2}$ Institute of Plant and Food Science, Southern University of Science and Technology, Shenzhen 518055, China. ${ }^{3}$ Key Laboratory of Molecular Design for Plant Cell Factory of Guangdong Higher Education Institutes, Southern University of Science and Technology, Shenzhen 518055, China. ${ }^{4}$ State Key Laboratory of Plant Genomics and National Center for Plant Gene Research (Beijing), Institute of Genetics and Developmental Biology, Chinese Academy of Sciences, Beijing 100101, China. 
Received: 24 July 2021 Accepted: 1 November 2021

Published online: 25 November 2021

\section{References}

1. Eaton JD, West S. Termination of transcription by RNA Polymerase II: BOOM! Trends Genet. 2020;36(9):664-75. https:// doi.org/10.1016/j.tig.2020.05.008

2. Proudfoot NJ. Transcriptional termination in mammals: stopping the RNA polymerase II juggernaut. Science. 2016; 352(6291). https://doi.org/10.1126/science.aad9926.

3. Porrua O, Libri D. Transcription termination and the control of the transcriptome: why, where and how to stop. Nat Rev Mol Cell Biol. 2015;16(3):190-202. https://doi.org/10.1038/nrm3943.

4. Logan J, Falck-Pedersen E, Darnell JE, Shenk T. A poly(A) addition site and a downstream termination region are required for efficient cessation of transcription by RNA polymerase II in the mouse beta maj-globin gene. Proc Natl Acad Sci. 1987:84(23):8306-10. https://doi.org/10.1073/pnas.84.23.8306.

5. Connelly S, Manley $\mathrm{J}$. A functional mRNA polyadenylation signal is required for transcription termination by RNA polymerase II. Genes Dev. 1988;2(4):440-52. https://doi.org/10.1101/gad.2.4.440.

6. Rosonina E. Terminating the transcript: breaking up is hard to do. Genes Dev. 2006;20(9):1050-6. https://doi.org/10.1101/ gad.1431606.

7. Proudfoot NJ. Ending the message: poly(A) signals then and now. Genes Dev. 2011;25(17):1770-82. https://doi.org/10.11 01/gad.17268411.

8. Luo W, Johnson AW, Bentley DL. The role of Rat1 in coupling mRNA 3'-end processing to transcription termination: implications for a unified allosteric-torpedo model. Genes Dev. 2006;20(8):954-65. https://doi.org/10.1101/gad.1409106.

9. West S, Gromak N, Proudfoot NJ. Human 5' $\rightarrow$ 3' exonuclease Xrn2 promotes transcription termination at cotranscriptional cleavage sites. Nature. 2004;432(7016):522-5. https://doi.org/10.1038/nature03035.

10. Lenstra TL, Rodriguez J, Chen H, Larson DR. Transcription dynamics in living cells. Annu Rev Biophys. 2016;45(1):25-47. https://doi.org/10.1146/annurev-biophys-062215-010838.

11. Nagarajan VK, Jones Cl, Newbury SF, Green PJ. XRN 5' $\rightarrow$ 3' exoribonucleases: Structure, mechanisms and functions. Biochim Biophys Acta BBA - Gene Regul Mech. 1829;2013(6-7):590-603. https://doi.org/10.1016/j.bbagrm.2013.03.005.

12. Duc C, Sherstnev A, Cole C, Barton GJ, Simpson GG. Transcription termination and chimeric RNA formation controlled by Arabidopsis thaliana FPA. PLoS Genet. 2013;9(10):e1003867. https://doi.org/10.1371/journal.pgen.1003867.

13. Eaton JD, Francis L, Davidson L, West S. A unified allosteric/torpedo mechanism for transcriptional termination on human protein-coding genes. Genes Dev. 2020;34(1-2):132-45. https://doi.org/10.1101/gad.332833.119.

14. Krzyszton M, Zakrzewska-Placzek M, Kwasnik A, Dojer N, Karlowski W, Kufel J. Defective XRN3-mediated transcription termination in Arabidopsis affects the expression of protein-coding genes. Plant J. 2018;93(6):1017-31. https://doi.org/1 $0.1111 /$ tpj.13826

15. Baejen C, Andreani J, Torkler P, Battaglia S, Schwalb B, Lidschreiber M, et al. Genome-wide analysis of RNA Polymerase ॥ termination at protein-coding genes. Mol Cell. 2017;66:38-49.e6.

16. Hazelbaker DZ, Marquardt S, Wlotzka W, Buratowski S. Kinetic competition between RNA polymerase II and Sen1dependent transcription termination. Mol Cell. 2013;49(1):55-66. https://doi.org/10.1016/j.molcel.2012.10.014.

17. Schwalb B, Michel M, Zacher B, Frühauf K, Demel C, Tresch A, et al. TT-seq maps the human transient transcriptome. Science. 2016;352(6290):1225-8. https://doi.org/10.1126/science.aad9841.

18. Fong N, Brannan K, Erickson B, Kim H, Cortazar MA, Sheridan RM, et al. Effects of transcription elongation rate and Xrn2 exonuclease activity on RNA polymerase II termination suggest widespread kinetic competition. Mol Cell. 2015;60(2): 256-67. https://doi.org/10.1016/j.molcel.2015.09.026.

19. Cheng C-Y, Krishnakumar V, Chan AP, Thibaud-Nissen F, Schobel S, Town CD. Araport11: a complete reannotation of the Arabidopsis thaliana reference genome. Plant J. 2017;89(4):789-804. https://doi.org/10.1111/tpj.13415.

20. Wissink EM, Vihervaara A, Tippens ND, Lis JT. Nascent RNA analyses: tracking transcription and its regulation. Nat Rev Genet. 2019;20(12):705-23. https://doi.org/10.1038/s41576-019-0159-6.

21. Core $L$, Waterfall JJ, Lis JT. Nascent RNA sequencing reveals widespread pausing and divergent initiation at human promoters. Science. 2008;322(5909):1845-8. https://doi.org/10.1126/science.1162228.

22. Kwak H, Fuda NJ, Core LJ, Lis JT. Precise maps of RNA polymerase reveal how promoters direct initiation and pausing. Science. 2013;339(6122):950-3. https://doi.org/10.1126/science.1229386.

23. Churchman LS, Weissman JS. Nascent transcript sequencing visualizes transcription at nucleotide resolution. Nature. 2011;469(7330):368-73. https://doi.org/10.1038/nature09652.

24. Nojima T, Gomes T, Grosso ARF, Kimura H, Dye MJ, Dhir S, et al. Mammalian NET-Seq reveals genome-wide nascent transcription coupled to RNA processing. Cell. 2015;161(3):526-40. https://doi.org/10.1016/j.cell.2015.03.027.

25. Sousa-Luís R, Dujardin G, Zukher I, Kimura H, Weldon C, Carmo-Fonseca M, et al. POINT technology illuminates the processing of polymerase-associated intact nascent transcripts. Mol Cell. 2021;81(9):1935-1950.e6. https://doi.org/10.101 6/j.molcel.2021.02.034.

26. van Dijk EL, Jaszczyszyn Y, Naquin D, Thermes C. The third revolution in sequencing technology. Trends Genet. 2018; 34(9):666-81. https://doi.org/10.1016/j.tig.2018.05.008.

27. Logsdon GA, Vollger MR, Eichler EE. Long-read human genome sequencing and its applications. Nat Rev Genet. 2020; 21(10):597-614. https://doi.org/10.1038/s41576-020-0236-x.

28. Legnini I, Alles J, Karaiskos N, Ayoub S, Rajewsky N. FLAM-seq: full-length mRNA sequencing reveals principles of poly(A) tail length control. Nat Methods. 2019;16(9):879-86. https://doi.org/10.1038/s41592-019-0503-y.

29. Liu Y, Nie H, Liu H, Lu F. Poly(A) inclusive RNA isoform sequencing (PAlso-seq) reveals wide-spread non-adenosine residues within RNA poly(A) tails. Nat Commun. 2019;10(1):1-13. https://doi.org/10.1038/s41467-019-13228-9.

30. Parker MT, Knop K, Sherwood AV, Schurch NJ, Mackinnon K, Gould PD, et al. Nanopore direct RNA sequencing maps the complexity of Arabidopsis mRNA processing and m6A modification. eLife. 2020;9:e49658. https://doi.org/10.7554/ elife.49658.

31. Roach NP, Sadowski N, Alessi AF, Timp W, Taylor J, Kim JK. The full-length transcriptome of C. elegans using direct RNA sequencing. Genome Res. 2020;30(2):299-312. https://doi.org/10.1101/gr.251314.119. 
32. Workman RE, Tang AD, Tang PS, Jain M, Tyson JR, Razaghi R, et al. Nanopore native RNA sequencing of a human poly(A) transcriptome. Nat Methods. 2019;16(12):1297-305. https://doi.org/10.1038/s41592-019-0617-2.

33. Drexler HL, Choquet K, Merens HE, Tang PS, Simpson JT, Churchman LS. Revealing nascent RNA processing dynamics with nano-COP. Nat Protoc. 2021;16(3):1343-75. https://doi.org/10.1038/s41596-020-00469-y.

34. Drexler HL, Choquet K, Churchman LS. Splicing kinetics and coordination revealed by direct nascent RNA sequencing through nanopores. Mol Cell. 2020;77:985-998.e8.

35. Reimer KA, Mimoso CA, Adelman K, Neugebauer KM. Co-transcriptional splicing regulates 3 ' end cleavage during mammalian erythropoiesis. Mol Cell. 2021;81 (5):998-1012.e7. https://doi.org/10.1016/.molcel.2020.12.018.

36. Herzel L, Straube K, Neugebauer KM. Long-read sequencing of nascent RNA reveals coupling among RNA processing events. Genome Res. 2018;28(7):1008-19. https://doi.org/10.1101/gr.232025.117.

37. Jia J, Long Y, Zhang H, Li Z, Liu Z, Zhao Y, et al. Post-transcriptional splicing of nascent RNA contributes to widespread intron retention in plants. Nat Plants. 2020;6(7):780-8. https://doi.org/10.1038/s41477-020-0688-1.

38. Long Y, Jia J, Mo W, Jin X, Zhai J. FLEP-seq: simultaneous detection of RNA polymerase II position, splicing status, polyadenylation site and poly(A) tail length at genome-wide scale by single-molecule nascent RNA sequencing. Nat Protoc. 2021;:1-27.

39. Hetzel J, Duttke SH, Benner C, Chory J. Nascent RNA sequencing reveals distinct features in plant transcription. Proc Natl Acad Sci. 2016;113(43):12316-21. https://doi.org/10.1073/pnas.1603217113.

40. Liu W, Duttke SH, Hetzel J, Groth M, Feng S, Gallego-Bartolome J, et al. RNA-directed DNA methylation involves cotranscriptional small-RNA-guided slicing of polymerase V transcripts in Arabidopsis. Nat Plants. 2018;4(3):181-8. https:// doi.org/10.1038/s41477-017-0100-y.

41. Zhu J, Liu M, Liu X, Dong Z. RNA polymerase II activity revealed by GRO-seq and pNET-seq in Arabidopsis. Nat Plants. 2018;4(12):1112-23. https://doi.org/10.1038/s41477-018-0280-0.

42. Kindgren $\mathrm{P}$, Ivanov $\mathrm{M}, \mathrm{Marquardt} \mathrm{S}$. Native elongation transcript sequencing reveals temperature dependent dynamics of nascent RNAPII transcription in Arabidopsis. Nucleic Acids Res. 2020;48(5):2332-47. https://doi.org/10.1093/nar/gkz11 89.

43. Di Giammartino DC, Nishida K, Manley JL. Mechanisms and consequences of alternative polyadenylation. Mol Cell. 2011; 43(6):853-66. https://doi.org/10.1016/j.molcel.2011.08.017.

44. Zhang S, Li R, Zhang L, Chen S, Xie M, Yang L, et al. New insights into Arabidopsis transcriptome complexity revealed by direct sequencing of native RNAs. Nucleic Acids Res. 2020;48(14):7700-11. https://doi.org/10.1093/nar/gkaa588.

45. Wu X, Liu M, Downie B, Liang C, Ji G, Li QQ, et al. Genome-wide landscape of polyadenylation in Arabidopsis provides evidence for extensive alternative polyadenylation. Proc Natl Acad Sci. 2011;108(30):12533-8. https://doi.org/10.1073/pna s.1019732108.

46. Lozano R, Booth GT, Omar BY, Li B, Buckler ES, Lis JT, et al. RNA polymerase mapping in plants identifies enhancers enriched in causal variants. bioRxiv. 2018;:376640.

47. Crisp PA, Smith AB, Ganguly DR, Murray KD, Eichten SR, Millar AA, et al. RNA Polymerase II read-through promotes expression of neighboring genes in SAL1-PAP-XRN retrograde signaling. Plant Physiol. 2018;178(4):1614-30. https://doi. org/10.1104/pp.18.00758.

48. Gy I, Gasciolli V, Lauressergues D, Morel J-B, Gombert J, Proux F, et al. Arabidopsis FIERY1, XRN2, and XRN3 are endogenous RNA silencing suppressors. Plant Cell. 2007;19(11):3451-61. https://doi.org/10.1105/tpc.107.055319.

49. Dye MJ, Proudfoot NJ. Multiple transcript cleavage precedes polymerase release in termination by RNA polymerase II. Cell. 2001;105(5):669-81. https://doi.org/10.1016/50092-8674(01)00372-5.

50. Cortazar MA, Sheridan RM, Erickson B, Fong N, Glover-Cutter K, Brannan K, et al. Control of RNA Pol II Speed by PNUTSPP1 and Spt5 dephosphorylation facilitates termination by a "sitting duck torpedo" mechanism. Mol Cell. 2019;76:896908.e4.

51. Chen FX, Smith ER, Shilatifard A. Born to run: control of transcription elongation by RNA polymerase II. Nat Rev Mol Cell Biol. 2018;19(7):464-78. https://doi.org/10.1038/s41580-018-0010-5.

52. Liu X, Kraus WL, Bai X. Ready, pause, go: regulation of RNA polymerase II pausing and release by cellular signaling pathways. Trends Biochem Sci. 2015;40(9):516-25. https://doi.org/10.1016/j.tibs.2015.07.003.

53. Liu C, Xin Y, Xu L, Cai Z, Xue Y, Liu Y, et al. Arabidopsis ARGONAUTE 1 binds chromatin to promote gene transcription in response to hormones and stresses. Dev Cell. 2018;44:348-361.e7.

54. Yu X, Martin PGP, Michaels SD. BORDER proteins protect expression of neighboring genes by promoting $3^{\prime}$ Pol II pausing in plants. Nat Commun. 2019;10(1):4359. https://doi.org/10.1038/s41467-019-12328-w.

55. Hornyik C, Terzi LC, Simpson GG. The Spen family protein FPA controls alternative cleavage and polyadenylation of RNA. Dev Cell. 2010;18(2):203-13. https://doi.org/10.1016/j.devcel.2009.12.009.

56. Parker MT, Knop K, Zacharaki V, Sherwood AV, Tomé D, Yu X, et al. Widespread premature transcription termination of Arabidopsis thaliana NLR genes by the spen protein FPA. eLife. 2021;10:e65537. https://doi.org/10.7554/eLife.65537.

57. Lu Z, Hofmeister BT, Vollmers C, DuBois RM, Schmitz RJ. Combining ATAC-seq with nuclei sorting for discovery of cisregulatory regions in plant genomes. Nucleic Acids Res. 2017;45(6):e41. https://doi.org/10.1093/nar/gkw1179.

58. Fan X, Moqtaderi Z, Jin Y, Zhang Y, Liu XS, Struhl K. Nucleosome depletion at yeast terminators is not intrinsic and can occur by a transcriptional mechanism linked to 3'-end formation. Proc Natl Acad Sci U S A. 2010;107(42):17945-50. https://doi.org/10.1073/pnas.1012674107.

59. White RJ. Transcription by RNA polymerase III: more complex than we thought. Nat Rev Genet. 2011;12(7):459-63. https://doi.org/10.1038/nrg3001.

60. Coulombe B, Burton ZF. DNA bending and wrapping around RNA polymerase: a "revolutionary" model describing transcriptional mechanisms. Microbiol Mol Biol Rev. 1999;63(2):457-78. https://doi.org/10.1128/MMBR.63.2.457-478.1999.

61. Li G, Liu S, Wang J, He J, Huang H, Zhang Y, et al. ISWI proteins participate in the genome-wide nucleosome distribution in Arabidopsis. Plant J. 2014;78(4):706-14. https://doi.org/10.1111/tpj.12499.

62. Liu Q, Bischof S, Harris CJ, Zhong Z, Zhan L, Nguyen C, et al. The characterization of Mediator 12 and 13 as conditional positive gene regulators in Arabidopsis. Nat Commun. 2020;1 1(1):2798. https://doi.org/10.1038/s41467-020-16651-5.

63. Martínez-Calvillo S, Nguyen D, Stuart K, Myler PJ. Transcription initiation and termination on Leishmania major Chromosome 3. Eukaryot Cell. 2004;3(2):506-17. https://doi.org/10.1128/EC.3.2.506-517.2004. 
64. Kurihara Y, Schmitz RJ, Nery JR, Schultz MD, Okubo-Kurihara E, Morosawa T, et al. Surveillance of $3^{\prime}$ noncoding transcripts requires FIERY1 and XRN3 in Arabidopsis. G3 Genes Genomes Genet. 2012;2:487-98.

65. Kankel MW, Ramsey DE, Stokes TL, Flowers SK, Haag JR, Jeddeloh JA, et al. Arabidopsis MET1 cytosine methyltransferase mutants. Genetics. 2003;163(3):1109-22. https://doi.org/10.1093/genetics/163.3.1109.

66. Li Q, Chen S, Leung AW-S, Liu Y, Xin Y, Zhang L, et al. DNA methylation affects pre-mRNA transcriptional initiation and processing in Arabidopsis, bioRxiv. 2021;:2021.04.29.441938.

67. Stroud H, Greenberg MVC, Feng S, Bernatavichute W, Jacobsen SE. Comprehensive analysis of silencing mutants reveals complex regulation of the Arabidopsis methylome. Cell. 2013;152(1-2):352-64. https://doi.org/10.1016/j.cell.2012.10.054.

68. Thomas QA, Ard R, Liu J, Li B, Wang J, Pelechano V, et al. Transcript isoform sequencing reveals widespread promoterproximal transcriptional termination in Arabidopsis. Nat Commun. 2020;11(1):2589. https://doi.org/10.1038/s41467-020-1 6390-7.

69. Glover-Cutter K, Kim S, Espinosa J, Bentley DL. RNA polymerase II pauses and associates with pre-mRNA processing factors at both ends of genes. Nat Struct Mol Biol. 2008;15(1):71-8. https://doi.org/10.1038/nsmb1352.

70. Wang $Y$, Wang $H, X i F$, Wang $H$, Han X, Wei W, et al. Profiling of circular RNA N6-methyladenosine in moso bamboo (Phyllostachys edulis) using nanopore-based direct RNA sequencing. J Integr Plant Biol. 2020;62(12):1823-38. https://doi. org/10.1111/jipb.13002.

71. Smith AM, Jain M, Mulroney L, Garalde DR, Akeson M. Reading canonical and modified nucleobases in 165 ribosomal RNA using nanopore native RNA sequencing. PLoS ONE. 2019;14(5):e0216709. https://doi.org/10.1371/journal.pone.021 6709.

72. Li H. Minimap2: pairwise alignment for nucleotide sequences. Bioinformatics. 2018;34(18):3094-100. https://doi.org/10.1 093/bioinformatics/bty191.

73. Li H, Handsaker B, Wysoker A, Fennell T, Ruan J, Homer N, et al. The Sequence Alignment/Map format and SAMtools. Bioinforma Oxf Engl. 2009;25(16):2078-9. https://doi.org/10.1093/bioinformatics/btp352.

74. Chen S, Zhou Y, Chen Y, Gu J. fastp: an ultra-fast all-in-one FASTQ preprocessor. Bioinformatics. 2018;34(17):i884-90. https://doi.org/10.1093/bioinformatics/bty560.

75. Xi Y, Li W. BSMAP: whole genome bisulfite sequence MAPping program. BMC Bioinformatics. 2009;10(1):1-9. https://doi. org/10.1186/1471-2105-10-232.

76. Dobin A, Davis CA, Schlesinger F, Drenkow J, Zaleski C, Jha S, et al. STAR: ultrafast universal RNA-seq aligner. Bioinformatics. 2013;29(1):15-21. https://doi.org/10.1093/bioinformatics/bts635.

77. Langmead B, Salzberg SL. Fast gapped-read alignment with Bowtie 2. Nat Methods. 2012;9(4):357-9. https://doi.org/10.1 038/nmeth.1923.

78. Ramírez F, Ryan DP, Grüning B, Bhardwaj V, Kilpert F, Richter AS, et al. deepTools2: a next generation web server for deep-sequencing data analysis. Nucleic Acids Res. 2016;44(W1):W160-5. https://doi.org/10.1093/nar/gkw257.

79. Zhang Y, Liu T, Meyer CA, Eeckhoute J, Johnson DS, Bernstein BE, et al. Model-based analysis of ChIP-Seq (MACS). Genome Biol. 2008;9(9):R137. https://doi.org/10.1186/gb-2008-9-9-r137.

80. Mo W, Liu B, Zhang H, Jin X, Lu D, Yu Y, et al. Zhai J. Genome Sequence Archive: Landscape of transcription termination in Arabidopsis revealed by single-molecule nascent RNA sequencing; 2021. https://ngdc.cncb.ac.cn/bioproject/browse/ PRJCA005821

81. Mo W, Liu B, Zhang H, Jin X, Lu D, Yu Y, et al. Zhai J. GitHub: Landscape of transcription termination in Arabidopsis revealed by single-molecule nascent RNA sequencing; 2021. https://github.com/ZhaiLab-SUSTech/Termination_landsca pe

82. Mo W, Liu B, Zhang $H$, Jin $X$, Lu D, Yu Y, et al. Landscape of transcription termination in Arabidopsis revealed by singlemolecule nascent RNA sequencing. Zenodo. 2021. https://doi.org/10.5281/zenodo.5607519.

\section{Publisher's Note}

Springer Nature remains neutral with regard to jurisdictional claims in published maps and institutional affiliations.

Ready to submit your research? Choose BMC and benefit from:

- fast, convenient online submission

- thorough peer review by experienced researchers in your field

- rapid publication on acceptance

- support for research data, including large and complex data types

- gold Open Access which fosters wider collaboration and increased citations

- maximum visibility for your research: over $100 \mathrm{M}$ website views per year

At $B M C$, research is always in progress.

Learn more biomedcentral.com/submissions 